\title{
Peter Derleder
}

\section{Der Erste bei der}

\section{verfassungsrechtlichen Überformung des Privatrechts}

\section{Thilo Ramm zum 85. Geburtstag}

Thilo Ramm war ein Solitär der Arbeitsrechtswissenschaft nach dem Zweiten Weltkrieg und ist bis heute ein Solitär geblieben, mit Ecken und Kanten, prononcierten Bekundungen von Respekt oder Desinteresse, Eigenwilligkeiten und Distanziertheit. Auch dem 85jährigen, der 1925 in Darmstadt geboren wurde, sieht man diese Mischung nicht an, da er stets einen offenen, wachen, freundlichen, ja charmanten Habitus behalten hat. Der früh verstorbene Vater war Angestellter eines weltberühmten Pharmaunternehmens, in dessen Auftrag er gelegentlich bis nach New York kam, mit gutem Englisch, insofern ein Vorbild. Fast wichtiger noch wurde ihm sein über ein Jahrzehnt älterer Bruder, der dem Schüler erste Instruktionen für eine wissenschaftliche Weise des Herangehens an Konflikte gab, aber noch vor Kriegende fiel. Alles überstrahlte aber die vitale Mutter, die vieles von ihm forderte, gefühlsmäßig die Nazis verachtete und auch den Sohn vor ideologischen Verwirrungen bewahrte.

Mit dem elterlichen Ehrgeiz war es verbunden, dass der Junge eine Klasse übersprang und schon mit neun in die Sekundarstufe kam. Das hatte entscheidende Bedeutung für sein weiteres Leben, weil er mit 17 Abitur machen konnte, nicht gleich eingezogen wurde und 1942/43 noch drei Semester Jura in Frankfurt und Marburg absolvieren konnte. An den elterlichen Bildungshaushalt knüpfte er mit der Lektüre der großen Historiker (Ranke, Treitschke u. a.) an. In Marburg 1943 war von vornherein Fritz von Hippel (Jahrgang 1897) seine Hauptbezugsperson im Unterricht wie in der Haltung. Dekan und dann auch Rektor der Universität Marburg war zu dieser Zeit Parteigenosse Rudolf Reinhardt, der vom Gauleiter empfohlen war. Als Thilo Ramm mit einem etwas nazikritischen Sketch auffiel, half ihm Reinhardt mit einer Unterbrechung des Studiums zugunsten des Fronteinsatzes, es hätte Schlimmeres kommen können. Den Rest des Krieges überstand der Soldat, wenn auch nicht ganz unverletzt.

In Anlehnung an seinen Vater hatte er sich guter Englischkenntnisse berühmt, weswegen ihn dann 1945 in Augsburg ein US-Oberstleutnant zum Übersetzer und Dolmetscher wählte. Von der Mutter, die zunächst in den Osten verschlagen war, war er schon länger getrennt und entwickelte sich als Angestellter der USMilitärregierung zu einer selbständigen Persönlichkeit, ein ansehnlicher Mann, mit immer besseren sprachlichen Kenntnissen, eigenständiger soziologischer Beobachtung des Entnazifizierungsdiskurses und ein Informationsvermittler zwischen der Besatzungsmacht und dem einer Erziehung zur Demokratie bedürftigen deutschen Volk. So half er kleinen Selbständigen, denen wegen Parteizugehörigkeit ein Betriebstreuhänder drohte, zu einer eigenständigen Selbstdarstellung jenseits des Fragebogens, da die Betriebe mit der Bezahlung eines Treuhänders überfordert gewesen wären. Außerdem nahm er an den Diskussionen über die Zukunft im Kalten Krieg auch unter den Militärs teil. 
Der Absprung fiel ihm nicht leicht, da die Versorgung beim US-Militär bestens war. 1946/47 studierte er dann die noch erforderlichen drei Semester wieder in Marburg, mit Anschluss wiederum an Fritz von Hippel. Die Naziprofessoren waren in dieser Zeit vorübergehend nicht aktiv. Viel lernen konnte man auch nicht, da zu viel Überlebensfragen auf der täglichen Agenda standen. Immerhin Erstes Staatsexamen 1947, Referendarausbildung bis 1950, mit einer kurzen Suspendierung wegen mangelnder Information über die Aktivitäten für die Militärregierung. Inzwischen wechselte Fritz von Hippel nach Freiburg, und Thilo Ramm folgte ihm, um bei ihm mit einem Stipendium zu promovieren.

Worüber würden Sie denn gern wissenschaftlich arbeiten? muss die vorbehaltlose Frage Fritz von Hippels gewesen sein. Nach all den Wirrungen der deutschen Geschichte, den Schrecken des Nationalsozialismus und den Gesprächen mit den Militärs kam der junge Wissenschaftler zu der Überzeugung, dass er sich über die sozialistischen Theorien informieren sollte. So entstand die Dissertation über Ferdinand Lasalle, die heute noch eine überzeugende, gut lesbare Darstellung bietet. Für einen Einstieg in das Privatrecht war das außerordentlich unkonventionell. Und wo er durch die Dissertation schon eingearbeitet war, lag es nahe, bei der Habilitation die Sache fortzusetzen, mit einem Werk über die großen Sozialisten des 19. Jahrhunderts, vor allem auch der nicht marxistischen. Zeitweise finanzierte er diese wissenschaftliche Arbeit mit einem Gehalt als Geschäftsführer der Gesellschaft zur Wahrung der Bürgerrechte, einer nach USVorbild gegründeten US-amerikanischen Bürgerrechtsgesellschaft, später dann mit einem Habilitationsstipendium. Wissenschaftlicher Assistent war er nie (abgesehen von einem Semester Tätigkeit als wissenschaftliche Hilfskraft zur Lebensmittelversorgung bei einem Rechtshistorikerkongress 1947). Fritz von Hippel ließ dem Nachwuchswissenschaftler freie Hand. Das Werk sollte in zwei Bänden erscheinen, von denen der zweite aber aus seiner heutigen Sicht unvollständig geblieben ist. Bei der Arbeit am ersten Band kam es auch zum Kontakt mit Wolfgang Abendroth, den Thilo Ramm aufgrund seiner gesellschaftlichen und politischen Kompetenz bewunderte. Nach und nach lernte er auch die bedeutenden Rechtsprofessoren unter den Emigranten kennen, Ernst Fraenkel, Franz L. Neumann und Otto Kahn-Freund (letzteren in London).

Eine kleine Bizarrerie der Nachkriegszeit ergab sich aus der Interpretation der hessischen Verfassung von 1946 und deren pazifistischem Geist. Während das Grundgesetz die Kriegsdienstverweigerung aus Gewissensgründen gewährleistete, leitete Ramm Anfang der 50er Jahre aus der hessischen Verfassung ein Verweigerungsrecht auch ohne Gewissensgründe her. Und Landesverfassungen konnten in ihrer Grundrechtsgeltung nach Art. 142 GG weitergehen, so dass der Wiederaufbau der Bundeswehr nur mit entsprechender regionaler Beschränkung hätte geschehen können. Das Amt Blank, das vor dem verteidigungspolitischen Auftritt von Franz Josef Strauß die Nazimilitärs in demokratische Soldaten umwandeln sollte, setzte sich jedoch über derart abseitige Äußerungen hinweg. Wer Anfang der 50er Jahre über Sozialisten geschrieben hatte, galt, auch wenn es sich um eine Darstellung handelte, automatisch selbst als Sozialist, was für Thilo Ramm jedoch keine self-fulfilling prophecy war. Die Habilitationsschrift war danach kein Selbstlauf. Es musste ein Paket mit drei Habilitanten geschnürt werden, damit Ramm die venia legendi für Rechtstheorie, Arbeitsrecht und Bürgerliches Recht erhalten konnte. Erik Wolf, ehemaliger Parteigenosse, aber auch Mitglied der Bekennenden Kirche, wollte auf seinem philosophisch-historischen Sektor an sich keine Konkurrenz. Im Arbeitsrecht sah die Sache anders aus, da die Naziarbeitsrechtler vielfach noch nicht wieder aktiv waren und Arbeitsrecht deswegen semesterlang in Freiburg nicht gelesen worden war. Die Probevorle- 
sung von 1953 ging über das Thema „Verfassung und Privatrecht“ und führte zu dem für die Gesamtheit der unter dem Nationalsozialismus ins Amt gekommenen oder von ihm erfassten Privatrechtler erschreckenden Ergebnis, dass die privatrechtliche Ordnung mit ihren Säulen Privateigentum und Vertragsfreiheit dringend der verfassungsrechtlichen Durchdringung bedurfte. Das war der Anstoß zu einem Prozess, der dann nach unendlich vielen Diskussionen zur mittelbaren Drittwirkung der Grundrechte und schließlich zur Rechtsprechung des Bundesverfassungsgerichts im Handelsvertreter- und im Bürgschaftsurteil führte. Damit stellte sich der 28-jährige Nachwuchswissenschaftler gegen die Phalanx der Privatrechtler, die nach dem „Zusammenbruch“ endlich ihre Ruhe vor der Politik in der frühliberalen Kodifikation von 1900 finden wollten. Die Antrittsvorlesung zur Anfechtung des Arbeitsvertrags bestätigte, dass hier ein Abweichler aktiv war, der die gesellschaftliche und historische Rolle des Rechts aufarbeiten wollte. An seiner Seite war seit den Freiburger Jahren seine Frau Renate, ebenfalls eine Juristin, mit der er noch heute eine glückliche, kindgesegnete Ehe führt und mit der er bei der Entwicklung von Rechtskundecurricula auch beruflich eng zusammenarbeitete, bevor sie später Professorin für Jugend- und Sozialrecht in Darmstadt wurde.

Der Arbeitsrechtler Thilo Ramm geriet nach seiner Habilitation jedoch bald in einen scharfen inhaltlichen Gegensatz zu der - trotz inhaltlicher Belastung aufgrund der Arbeiten zum Naziarbeitsrecht - beherrschenden Figur des neugeordneten Arbeitsrechts der Adenauerzeit, Hans-Carl Nipperdey. Dieser schuf mit einem großen Stab wissenschaftlicher Mitarbeiter (zeitweise sollen es 64 gewesen sein) und Schüler und in seiner Rolle als erster BAG-Präsident (mit der heute unvorstellbaren autoritativen Einzelzuweisung der Arbeitsrechtssachen an geeignete Mitglieder des Gerichts) ein Arbeitsrecht, das eher den Anforderungen einer zügigen Kapitalverwertung als einer sozialen Dämpfung gerecht werden sollte. Der sog. rheinische Kapitalismus musste den Arbeitgebern und den Gerichten in Wahrheit erst von preußischen Sozialdemokraten wie Adolf Arndt mühsam abgerungen werden. In dieser Richtung wirkte auch Thilo Ramm, der nunmehr mit regelmäßigen Anmerkungen und Rezensionen in der Juristenzeitung die Schwachpunkte des Nipperdey'schen Arbeitsrechts aufspießte. Einem solchen Wissenschaftler konnte man keine Karriereaussichten bescheinigen.

Adolf Arndt, der später sog. Kronjurist der SPD, suchte denn auch Ende der 50er Jahre verzweifelt nach einem kritischen Arbeitsrechtler, der ein Gutachten machen sollte, um den Millionenschadensersatzforderungen gegen die Gewerkschaften nach dem schleswig-holsteinischen Metallarbeiterstreik 1956/57 entgegenzutreten. Dieses Gutachten wurde ein Teil der kontinuierlichen Arbeit Ramms zum Kollektivarbeitsrecht, dessen verfassungsrechtliche Gewährleistung nunmehr gegen die deliktische Qualifikation des Streiks umgesetzt werden musste. Das BAG entschied dem Grunde nach zu Lasten der Gewerkschaften. Der Hauptkassierer der IG Metall wollte zum großen Bedauern Ramms nicht auch noch ein Verfassungsbeschwerdeverfahren finanzieren. Aber zu den großen Schadensersatzzahlungen kam es dann um des lieben sozialen Friedens willen auch nicht.

In der Zusammenarbeit mit Sozialdemokraten und Gewerkschaftern war Thilo Ramm objektiv ein Linker geworden, auch wenn er sich selbst niemals als solcher gesehen hat. Bestraft wurde er von der herrschenden Zivilrechtswissenschaft dadurch, dass er keine Professur erhielt. Er war ein Jahrzehnt lang Privatdozent und schließlich außerplanmäßiger Professor, konnte aber von den Lehrgeldern aus gut besuchten Veranstaltungen leben. Erst mit der Neugründung von Universitäten wurde dieses Kapitel beendet. 
In Gießen war 1945 die Universität geschlossen worden, weil sie zerstört war. Georg August Zinn wollte sie wieder begründen, mit endlich unbelasteten jungen Professoren. Thilo Ramm wurde auf einen politikwissenschaftlichen Lehrstuhl berufen, da keine juristische Fakultät ihm trotz seiner herausragenden wissenschaftlichen Qualifikation einen Ruf erteilt hatte. Er musste nunmehr öffentliches Recht und Politologie lehren. Bei seiner Antrittsvorlesung beging er das Sakrileg, am Ende seiner Normanalyse Verhandlungen zwischen den beiden deutschen Staaten zu empfehlen, was selbst die SPD damals furchtbar erschreckte.

Dennoch keimte einige Zeit später im Gespräch mit dem Ministerpräsidenten Zinn dann die Idee einer juristischen Fakultät auf, die auch relativ schnell 1965 verwirklicht wurde. Als erster wurde Spiros Simitis berufen. Eine Reihe vielversprechender Reformer sollte gewonnen werden, für eine Universität ohne Altlast, mit Studienreformen und eventuell sogar Studenten aus der Arbeiterklasse. Thilo Ramm wechselte auf einen Lehrstuhl, der seiner venia entsprach. Helmut Ridder kam von Bonn nach Gießen, Walter Mallmann aus Frankfurt. Aus Tübingen kam Friedrich Kübler, aber nicht der heiß umworbene Strafrechtler Baumann. Thilo Ramm plante eine Reformfakultät, wie es im Mittelalter Cluny für die Theologie gewesen war. Manche Reformidee ging in die Vorstellungen der Frankfurter Wiethölter und Denninger ein oder wurde von diesen übernommen, von der Kleingruppenarbeit über die Verbindung von Theorie und Praxis bis zur gesellschaftlichen Analyse der Rechtsbestände. Pech war natürlich, dass Gießen doch ein wenig kosmopolitischen Charmes entbehrte und es deswegen auch zur Abwanderung der meisten zunächst Berufenen kam. Ramm selbst entwickelte aber in Gießen ein neues Eros als Lehrer, wie sich dies insbesondere in seiner Einführung in das Privatrecht niederschlug.

Aber dann kam 1968 mit der Folge, dass der hochverdiente kritische Rechtswissenschaftler Thilo Ramm von seinen Assistenten und Mitarbeitern links überholt wurde. Manche erarbeiteten sich marxistische Theorie so schnell, dass Ramm sie als Gefühlsmarxisten empfand, wo er doch selbst viele Jahre zur Aufarbeitung der Theorien des 19. Jahrhunderts gebraucht hatte. Er war gekränkt darüber, dass sie sich an ihm als Autorität rebellisch abarbeiteten, ohne seine besondere Rolle in den 50- und 60er Jahren nachzuvollziehen. Die schnell proklamierte Einheit von Theorie und Praxis wurde vor allem auf die Universität bezogen und konnte zivilisatorische Verstöße einschließen, bis hin zu Blockaden von Lehrveranstaltungen und Gremien. Dabei war Ramms näherer Umkreis durchaus eine Elite mit Namen wie Bernhard Weller, Manfred Weiß, Ulrich Mückenberger, Roderich Wahsner und den Assistenten Ridders. Es entstand eine große Kränkung, die heute noch nicht ganz verwunden ist, auch wenn die Jüngeren mit einer Spur von schlechtem Gewissen an diese Zeit zurückdenken dürften. Das Paradox war, dass ein Linker, der sich selbst nicht als Linker (sondern als jederzeit unabhängiger Wissenschaftler) verstand, doch dadurch verletzt war, dass er links überholt wurde.

Er war schon einverstanden damit, dass die arbeitsrechtliche Linke mit Wolfgang Däubler als Protagonisten an seine Vorarbeiten anknüpfte, verlangte von ihr aber mehr. Er wollte in rechtsdogmatischer Hinsicht nicht das gleiche Arbeitsrecht, „nur andersherum“, sondern eine „Vision“, was aber der gesellschaftlichen Situation der 70er Jahre letztlich nicht entsprach. Bei seiner Abrechnung mit den 68ern kam dann vieles in einen Topf, von der RAF bis zu den Unhöflichkeiten im akademischen Kontakt. Ramm hat dann auch nicht mehr zur Kenntnis genommen, wie viel die ehemaligen Gießener Nachwuchswissenschaftler in ihrer späteren Lehr- und Justizpraxis von ihm selbst übernommen haben. 
Das gilt nicht für die Methode, wo Thilo Ramm sich noch heute als Positivist versteht, der von einem Gesetzgeber die Lösungen erwartet, obwohl dieser sich doch weitgehend auf das Abschreiben von kompromisshaften Rechtsprechungsgrundsätzen und europäischen Richtlinien beschränkt. Auch für das Vorverständnis der Norminterpretation, das nach der Mehrzahl der Methodologen wenigstens aufgedeckt werden muss, hat Thilo Ramm nur Abwehrreflexe entwickelt. Aber in den Ergebnissen zum Verhältnis von Verfassungsrecht, Zivilrecht und Arbeitskampfrecht hat er, genau besehen, eine breite Nachfolge auch bei den früheren Gießenern gefunden. Die Kommunikationsstörung hatte praktisch langfristig mehr persönliche als inhaltliche Dimensionen.

Nach dem Konflikt mit den 68ern wich Thilo Ramm in den folgenden Jahren in das Familien- und Jugendrecht aus. Sein zweibändiges Taschenbuch zum Familienrecht war zeitweilig das aufgeklärteste, modernste und beste Lehrbuch, auch wenn dort immer nur vom Gleichberechtigungsgrundsatz und nie vom Patriarchat die Rede war. Aber er hat dann nicht nachgesetzt, was im Hinblick auf die revolutionäre Entwicklung dieses Rechtsgebiets bald nötig gewesen wäre. Das umfassende Lehrbuch zum Jugendrecht im Beck-Verlag von 1990, eine großartige Sammlungsleistung und Durchdringung eines facettenreichen Rechtsgebiets, auch unter Einbeziehung des Rechts der DDR, brach ihm durch die Wiedervereinigung weg. Für eine Neuauflage mit ganz anderem Konzept hatte er an der Pensionsgrenze nicht die Kraft.

Im Ruhestand ist Thilo Ramm ein ambitionierter Intellektueller geblieben. Er hat sich in Darmstadt, das aber nach den großen Kriegszerstörungen nicht mehr seine Kindheitsstadt ist, mehr auf die allgemeine Gesellschaftsgeschichte ausgerichtet. Er streitet noch gern um die filigranen Dimensionen wissenschaftlicher Begriffe, lässt sich auch von anderen Sichtweisen noch anregen. Er hat die historische Chance zur Bildung einer Schule seit den 50er Jahren nicht recht genutzt, die ihm mit der Folgegeneration langfristig möglich gewesen wäre. Das Drama seiner Begabung bestand darin, dass er die von ihm erarbeiteten rechtlichen Ideen und Konzepte gern beiseite legte, wenn sie einmal formuliert waren, in Verkennung der langwierigen Durchsetzungsprozesse des juristischen Sektors. Er ist sich aber auf höchst eigentümliche Weise insofern treu geblieben, als er stets die Distanz des Wissenschaftlers praktiziert hat, zur restaurativen Adenauerzeit wie zu den Reformideen von 1968, zur Kritischen Theorie wie zu den sozialen Bewegungen. „Wie es wirklich gewesen ist“, die Passion, das zu erkennen, erfüllt ihn noch heute, auch wenn er die von ihm beanspruchte begriffliche und theoretische Autonomie oft eher provozierend als selbstkritisch wahrnimmt. In jedem Fall verdient Thilo Ramm in einer Geschichte der kritischen Rechtswissenschaft nach 1950 den Rang eines Gründers. 